\title{
Extremal Functions for Trudinger-Moser Type Inequalities in $\mathbb{R}^{N}$
}

\section{Xiaomeng ${ }^{1,2, *}$}

${ }^{1}$ School of Information, Renmin University of China, Beijing 100872, China.

${ }^{2}$ School of Information, Huaibei Normal University, Huaibei 235000, China.

Received 11 October 2016; Accepted 8 January 2017

Abstract. Let $N \geq 2, \alpha_{N}=N \omega_{N-1}^{1 /(N-1)}$, where $\omega_{N-1}$ denotes the area of the unit sphere in $\mathbb{R}^{N}$. In this note, we prove that for any $0<\alpha<\alpha_{N}$ and any $\beta>0$, the supremum

$$
\sup _{u \in W^{1, N}\left(\mathbb{R}^{N}\right),\|u\|_{W^{1, N}\left(\mathbb{R}^{N}\right)} \leq 1} \int_{\mathbb{R}^{N}}|u|^{\beta}\left(e^{\alpha|u|^{\frac{N}{N-1}}}-\sum_{j=0}^{N-2} \frac{\alpha^{j}}{j !}|u|^{N-1}\right) \mathrm{d} x
$$

can be attained by some function $u \in W^{1, N}\left(\mathbb{R}^{N}\right)$ with $\|u\|_{W^{1, N}\left(\mathbb{R}^{N}\right)}=1$. Moreover, when $\alpha \geq \alpha_{N}$, the above supremum is infinity.

AMS Subject Classifications: 46E35

Chinese Library Classifications: O175.29

Key Words: Extremal function; Trudinger-Moser inequality.

\section{Introduction and main results}

Let $N \geq 2$ and $\alpha_{N}=N \omega_{N-1}^{1 /(N-1)}$, where $\omega_{N-1}$ is the area of the unit sphere in $\mathbb{R}^{N}$. For any bounded domain $\Omega \subset \mathbb{R}^{N}$, we denote $W_{0}^{1, N}(\Omega)$ the closure of $C_{0}^{\infty}(\Omega)$ under the norm

$$
\|u\|_{W_{0}^{1, N}(\Omega)}=\left(\int_{\Omega}|\nabla u|^{N} \mathrm{~d} x\right)^{1 / N} .
$$

The classical Trudinger-Moser inequality [1-5] says

$$
\sup _{u \in W_{0}^{1, N}(\Omega),\|u\|_{W_{0}^{1, N}(\Omega)} \leq 1} \int_{\Omega} e^{\alpha_{N}|u|^{N /(N-1)}} \mathrm{d} x<\infty .
$$

${ }^{*}$ Corresponding author. Email address: xmlimath@ruc. edu. cn (X. M. Li) 
Define a function $\zeta: \mathbb{N} \times \mathbb{R} \rightarrow \mathbb{R}$ by

$$
\zeta(N, t)=e^{t}-\sum_{j=0}^{N-2} \frac{t^{j}}{j !}=\sum_{j=N-1}^{\infty} \frac{t^{j}}{j !} .
$$

The inequality (1.1) was extended by Cao [6], Panda [7], do Ó [8], Adachi-Tanaka [9] to the whole $\mathbb{R}^{N}$, namely

$$
\sup _{u \in W^{1, N}\left(\mathbb{R}^{N}\right),\|u\|_{W^{1, N\left(\mathbb{R}^{N}\right)} \leq 1}} \int_{\mathbb{R}^{N}} \zeta\left(N, \alpha|u|^{\frac{N}{N-1}}\right) \mathrm{d} x<\infty, \quad \forall 0<\alpha<\alpha_{N},
$$

where

$$
\|u\|_{W^{1, N}\left(\mathbb{R}^{N}\right)}=\left(\int_{\mathbb{R}^{N}}\left(|\nabla u|^{N}+|u|^{N}\right) \mathrm{d} x\right)^{1 / N} .
$$

The critical case of (1.2), $\alpha=\alpha_{N}$, was obtained by Ruf [10] and Li-Ruf [11]. Later, using the Young inequality, Adimurthi-Yang [12] provided a very simple proof of the critical Trudinger-Moser inequality in $\mathbb{R}^{N}$, as well as the singular Trudinger-Moser inequality. One of conclusions in [12] is that the inequality

$$
\int_{\mathbb{R}^{N}} \frac{\zeta\left(N, \alpha|u|^{\frac{N}{N-1}}\right)}{|x|^{\beta}} \mathrm{d} x<\infty,
$$

holds for any $\alpha>0,0 \leq \beta<N$ and any $u \in W^{1, N}\left(\mathbb{R}^{N}\right)(N \geq 2)$.

It was proved by Ruf [10] and Ishiwata [13] that the supremum

$$
\sup _{u \in W^{1,2}\left(\mathbb{R}^{2}\right),\|u\|_{W^{1,2}\left(\mathbb{R}^{2}\right)} \leq 1} \int_{\mathbb{R}^{2}}\left(e^{\alpha u^{2}}-1\right) \mathrm{d} x,
$$

can be attained when $\alpha^{*} \leq \alpha<4 \pi$ for some constant $\alpha^{*}>0$, and can not be attained when $0<\alpha \ll 1$. In the case $\alpha=\alpha_{N}$ and $N \geq 3$, the existence of extremal functions for the supremum in (1.2) was obtained by Li-Ruf [11]; while in the case $0<\alpha<\alpha_{N}$, the existence result was proved by Ishiwata [13].

From now on, we assume $N \geq 2$. In this note, we first prove a Trudinger-Moser type inequality, namely

Theorem 1.1. (i) For any $\beta>0$, any $\alpha>0$ and any $u \in W^{1, N}\left(\mathbb{R}^{N}\right)$, there holds

$$
\int_{\mathbb{R}^{N}}|u|^{\beta} \zeta\left(N, \alpha|u|^{N-1}\right) \mathrm{d} x<\infty .
$$

(ii) For any $\beta>0$ and any $0<\alpha<\alpha_{N}$, we have

$$
\sup _{u \in W^{1, N}\left(\mathbb{R}^{N}\right),\|u\|_{W^{1, N}\left(\mathbb{R}^{N}\right)} \leq 1} \int_{\mathbb{R}^{N}}|u|^{\beta} \zeta\left(N, \alpha|u|^{\frac{N}{N-1}}\right) \mathrm{d} x<\infty .
$$

(iii) For any $\beta>0$ and any $\alpha \geq \alpha_{N}$, the above supremum is infinity. 
Concerning the extremal function for (1.4), we have the following:

Theorem 1.2. For any $\beta>0$ and any $0<\alpha<\alpha_{N}$, the supremum

$$
\sup _{u \in W^{1, N}\left(\mathbb{R}^{N}\right),\|u\|_{W^{1, N\left(\mathbb{R}^{N}\right)}} \leq 1} \int_{\mathbb{R}^{N}}|u|^{\beta} \zeta\left(N, \alpha|u|^{\frac{N}{N-1}}\right) \mathrm{d} x
$$

can be attained by some function $u \in W^{1, N}\left(\mathbb{R}^{N}\right)$ with $\|u\|_{W^{1, N}\left(\mathbb{R}^{N}\right)}=1$.

Remark 1.1. In Theorems 1.1 and 1.2, if $|u|^{\beta}$ is replaced by a general term $\phi\left(|u|^{\beta}\right)$, where $\phi:[0, \infty) \rightarrow[0, \infty)$ is an increasing function satisfying

$$
\lim _{\alpha \rightarrow 0^{+}} \limsup _{s \rightarrow+\infty} \frac{\phi(s)}{\zeta\left(N, \alpha s^{\frac{N}{N-1}}\right)}<+\infty,
$$

then the same conclusion still holds.

Our proof of Theorems 1.1 and 1.2 is based on the Hölder inequality, the TrudingerMoser inequality (1.2) and concentration-compactness analysis. Before ending the introduction, we mention Carleson-Chang [14], Flucher [15], Lin [16], Li [17], Yang [18-20], Lu-Yang [21], Wang-Ye [22] and Yang-Zhu [23, 24] for existence of extremal functions for Trudinger-Moser inequalities on bounded Euclidean domain or compact Riemannian surface.

The remaining part of this note is organized as follows: Section 2 gives some preliminary results, and we prove Theorem 1.1 in Section 3. Finally, Theorem 1.2 will be proved in Section 4.

\section{Preliminaries}

In this section, we first introduce an elementary inequality due to Yang [25]. It would bring a great convenience during our calculation. Namely,

Lemma 2.1. Let $t \geq 0$ and $p \geq 1$. Then for any integer $N \geq 2$,

$$
(\zeta(N, t))^{p} \leq \zeta(N, p t) .
$$

We next recall several definitions on the Schwarz rearrangement [26]. Let $\Omega$ be a bounded smooth domain in $\mathbb{R}^{N}$ and $u$ be a nonnegative function belonging to $W_{0}^{1, N}(\Omega)$. $\mathbb{B}_{R}$ represents a ball of radius $R$ centered at the origin. $|\Omega|$ is the volume of $\Omega$. Define a function $u^{*}: \mathbb{B}_{R} \rightarrow \mathbb{R}$ satisfying:

(a) $\left|\mathbb{B}_{R}\right|=|\Omega|$;

(b) $\left|\left\{x \in \mathbb{B}_{R}: u^{*}(x)>t\right\}\right|=|\{x \in \Omega: u(x)>t\}|$ for $t>0$. 
Then $u^{*}$ is called the Schwarz rearrangement of $u . u^{*}$ is a decreasing radially symmetric function. Suppose $H: \mathbb{R} \rightarrow \mathbb{R}$ is a continuous and increasing function. By the rearrangement, one gets

$$
\int_{\mathbb{B}_{R}} H\left(u^{*}(x)\right) \mathrm{d} x=\int_{\Omega} H(u(x)) \mathrm{d} x, \quad \int_{\mathbb{B}_{R}}\left|\nabla u^{*}\right|^{N} \mathrm{~d} x \leq \int_{\Omega}|\nabla u|^{N} \mathrm{~d} x .
$$

For the whole Euclidean space $\mathbb{R}^{N}$, the Schwarz rearrangement can be similarly defined. For each $t \geq 0$, let $\chi_{\{u>t\}^{*}}$ be the characteristic function of the set $\{u>t\}^{*}=\mathbb{B}_{R}$, where $\left|\mathbb{B}_{R}\right|=|\{u>t\}|$. Define the Schwarz rearrangement of $u$ by

$$
u^{*}(x)=\int_{0}^{\infty} \chi_{\{u>t\}^{*}}(x) \mathrm{d} t
$$

Thus $u^{*}$ is a decreasing radially symmetric function. In addition, $u^{*}$ satisfies

$$
\int_{\mathbb{R}^{N}} H\left(u^{*}(x)\right) \mathrm{d} x=\int_{\mathbb{R}^{N}} H(u(x)) \mathrm{d} x, \quad \int_{\mathbb{R}^{N}}\left|\nabla u^{*}\right|^{N} \mathrm{~d} x \leq \int_{\mathbb{R}^{N}}|\nabla u|^{N} \mathrm{~d} x .
$$

Throughout this note, $\mathbb{B}_{R}$ denotes a ball centered at the origin with radius $R$; while $\mathbb{B}_{R}^{c}$ denotes its complement. $o_{n}(1) \rightarrow 0$ as $n \rightarrow \infty$. We often denote various constants by the same $C$. The reader can easily recognize it from the context. Moreover, we do not distinguish sequence and subsequence during the analysis.

\section{Proof of Theorem 1.1}

In this section, we prove Theorem 1.1. The proof of the first two parts of Theorem 1.1 is based on the Hölder inequality, Lemma 2.1, the Sobolev embedding theorem, the Trudinger-Moser inequality (1.2) and the inequality (1.3). The third part of Theorem 1.1 will be proved by a computation of the Moser function sequence.

Proof of Theorem 1.1 For any $\beta>0$, any $\alpha>0$ and any $u \in W^{1, N}\left(\mathbb{R}^{N}\right)$, we have by the Hölder inequality and Lemma 2.1,

$$
\begin{aligned}
\int_{\mathbb{R}^{N}}|u|^{\beta} \zeta\left(N, \alpha|u|^{\frac{N}{N-1}}\right) \mathrm{d} x & \leq\left(\int_{\mathbb{R}^{\mathbb{N}}}|u|^{\beta p} \mathrm{~d} x\right)^{1 / p}\left(\int_{\mathbb{R}^{N}}\left(\zeta\left(N, \alpha|u|^{\frac{N}{N-1}}\right)\right)^{p^{\prime}} \mathrm{d} x\right)^{1 / p^{\prime}} \\
& \leq\left(\int_{\mathbb{R}^{N}}|u|^{\beta p} \mathrm{~d} x\right)^{1 / p}\left(\int_{\mathbb{R}^{N}} \zeta\left(N, \alpha p^{\prime}|u|^{\frac{N}{N-1}}\right) \mathrm{d} x\right)^{1 / p^{\prime}} \\
& \leq C\left(\int_{\mathbb{R}^{N}} \zeta\left(N, \alpha p^{\prime}|u|^{\frac{N}{N-1}}\right) \mathrm{d} x\right)^{1 / p^{\prime}}
\end{aligned}
$$

for some constant $C$ only depending on $N, \beta, p$, where $1 / p+1 / p^{\prime}=1$ and $p>\max \{1, N / \beta\}$. Notice that the integral on the left-hand side of (3.1) is the special case of (1.3), namely $\beta=0$. Thus, the proof of $(i)$ is complete. 
Assume $0<\alpha<\alpha_{N}$ and $u \in W^{1, N}\left(\mathbb{R}^{N}\right)$ with $\|u\|_{W^{1, N}\left(\mathbb{R}^{N}\right)} \leq 1$. Choose $q^{\prime}>1$ sufficiently close to 1 such that $\alpha q^{\prime}<\alpha_{N}$. Again using the Hölder inequality and Lemma 2.1, we obtain

$$
\begin{aligned}
\int_{\mathbb{R}^{N}}|u|^{\beta} \zeta\left(N, \alpha|u|^{\frac{N}{N-1}}\right) \mathrm{d} x & \leq\left(\int_{\mathbb{R}^{\mathbb{N}}}|u|^{\beta q} \mathrm{~d} x\right)^{1 / q}\left(\int_{\mathbb{R}^{N}}\left(\zeta\left(N, \alpha|u|^{\frac{N}{N-1}}\right)\right)^{q^{\prime}} \mathrm{d} x\right)^{1 / q^{\prime}} \\
& \leq\left(\int_{\mathbb{R}^{N}}|u|^{\beta q} \mathrm{~d} x\right)^{1 / q}\left(\int_{\mathbb{R}^{N}} \zeta\left(N, \alpha q^{\prime}|u|^{N-1}\right) \mathrm{d} x\right)^{1 / q^{\prime}},
\end{aligned}
$$

where $1 / q+1 / q^{\prime}=1$. The two integrals on the left-hand side of (3.2) are both bounded, thanks to (1.2) and the Sobolev embedding theorem. Thus, the proof of (ii) is complete.

Finally, we prove for any $\alpha \geq \alpha_{N}$,

$$
\sup _{u \in W^{1, N}\left(\mathbb{R}^{N}\right),\|u\|_{W^{1, N}\left(\mathbb{R}^{N}\right)} \leq 1} \int_{\mathbb{R}^{N}}|u|^{\beta} \zeta\left(N, \alpha|u|^{\frac{N}{N-1}}\right) \mathrm{d} x=\infty .
$$

Recall Moser's function sequence,

$$
m_{n}(x, r)=\frac{1}{\omega_{N-1}^{1 / N}} \begin{cases}(\log n)^{(N-1) / N}, & |x| \leq r / n \\ (\log n)^{-1 / N} \log (r /|x|), & r / n<|x| \leq r \\ 0, & |x|>r .\end{cases}
$$

By straightforward calculation, we have

$$
\begin{aligned}
& \int_{\mathbb{R}^{N}}\left|\nabla m_{n}(x, r)\right|^{N} \mathrm{~d} x=\frac{1}{\omega_{N-1}} \int_{\frac{r}{n} \leq x \leq r} \frac{1}{(\log n)|x|^{N}} \mathrm{~d} x=1, \\
& \int_{|x| \leq \frac{r}{n}}\left|m_{n}(x, r)\right|^{N} \mathrm{~d} x=\frac{1}{\omega_{N-1}} \int_{|x| \leq \frac{r}{n}}(\log n)^{N-1} \mathrm{~d} x=\left(\frac{r}{n}\right)^{N} \frac{(\log n)^{N-1}}{N}=o_{n}(1) .
\end{aligned}
$$

Integration by parts, it follows that

$$
\begin{aligned}
& \int_{\frac{r}{n} \leq|x| \leq r}\left|m_{n}(x, r)\right|^{N} \mathrm{~d} x \\
= & \frac{1}{\log n} \int_{\frac{r}{n}}^{r} t^{N-1}\left(\log \frac{r}{t}\right)^{N} \mathrm{~d} t \\
= & -\frac{1}{N}\left(\frac{r}{n}\right)^{N}(\log n)^{N-1}+\frac{1}{\log n} \int_{\frac{r}{n}}^{r} t^{N-1}\left(\log \frac{r}{t}\right)^{N-1} \mathrm{~d} t \\
= & -\left(\frac{r}{n}\right)^{N}\left\{\frac{1}{N}(\log n)^{N-1}+\frac{1}{N}(\log n)^{N-2}+\frac{N-1}{N^{2}}(\log n)^{N-3}\right. \\
& \left.\quad+\cdots+\frac{(N-1)(N-2) \cdots 3}{N^{N-2}} \log n\right\}+\frac{1}{\log n} \frac{(N-1) !}{N^{N-2}} \int_{\frac{r}{n}}^{r} t^{N-1}\left(\log \frac{r}{t}\right) \mathrm{d} t \\
= & O_{n}(1) .
\end{aligned}
$$


Combining (3.5) and (3.6), we get

$$
\begin{aligned}
\int_{\mathbb{R}^{N}}\left|m_{n}(x, r)\right|^{N} \mathrm{~d} x & =\frac{1}{\omega_{N-1}} \int_{|x| \leq \frac{r}{n}}(\log n)^{N-1} \mathrm{~d} x+\frac{1}{\omega_{N-1}} \int_{\frac{r}{n} \leq|x| \leq r} \frac{\left(\log \frac{r}{|x|}\right)^{N}}{\log n} \mathrm{~d} x \\
& =o_{n}(1) .
\end{aligned}
$$

In view of (3.4) and (3.7), we obtain

$$
\left\|m_{n}(x, r)\right\|_{W^{1, N}\left(\mathbb{R}^{N}\right)}^{N}=\int_{\mathbb{R}^{N}}\left|m_{n}\right|^{N} \mathrm{~d} x+\int_{\mathbb{R}^{N}}\left|\nabla m_{n}\right|^{N} \mathrm{~d} x=1+o_{n}(1) .
$$

Considering $\widetilde{m}_{n}(x, r)=m_{n}(x, r) /\left\|m_{n}(x, r)\right\|_{W^{1, N}\left(\mathbb{R}^{N}\right)}$, it follows that

$$
\begin{aligned}
\int_{\mathbb{R}^{N}}\left|\widetilde{m}_{n}\right|^{\beta} \zeta\left(N, \alpha \widetilde{m}_{n}^{\frac{N}{N-1}}\right) \mathrm{d} x & \geq \int_{|x| \leq \frac{r}{n}}\left|\widetilde{m}_{n}\right|^{\beta}\left(e^{\alpha \widetilde{m}_{n}^{\frac{N}{N-1}}}-\sum_{j=0}^{N-2} \frac{\alpha^{j}}{j !} \widetilde{m}_{n}^{\frac{N}{N-1} j}\right) \mathrm{d} x \\
& \geq\left(n^{\frac{\alpha}{\omega_{N-1}^{1(N-1)}}} e^{O(1)}+O\left((\log n)^{N-2}\right)\right) \frac{\omega_{N-1}^{1-\frac{\beta}{N}} r^{N}(\log n)^{\frac{N-1}{N} \beta}}{\left(1+o_{n}(1)\right) N n^{N}} .
\end{aligned}
$$

For any $\alpha \geq \alpha_{N}$, we have

$$
\frac{(\log n)^{\frac{N-1}{N} \beta} \cdot n^{\frac{\alpha}{\omega_{N-1}^{1(N-1)}}}}{n^{N}} \geq(\log n)^{\frac{N-1}{N} \beta} \rightarrow \infty, \quad(n \rightarrow \infty) .
$$

Thus (3.3) holds and therefore we complete the proof of the Theorem 1.1.

\section{Proof of Theorem 1.2}

In this section, we will apply concentration-compactness analysis to obtain the existence of extremal function for supremum in (1.4).

Proof of Theorem 1.2 For any $u \in W^{1, N}\left(\mathbb{R}^{N}\right)$, we let $u^{*}$ be the Schwarz rearrangement of $|u|$, then

$$
\begin{aligned}
& \int_{\mathbb{R}^{N}}\left|u^{*}\right|^{N} \mathrm{~d} x=\int_{\mathbb{R}^{N}}|u|^{N} \mathrm{~d} x, \quad \int_{\mathbb{R}^{N}}\left|\nabla u^{*}\right|^{N} \mathrm{~d} x \leq \int_{\mathbb{R}^{N}}|\nabla u|^{N} \mathrm{~d} x, \\
& \int_{\mathbb{R}^{N}}\left|u^{*}\right|^{\beta} \zeta\left(N, \alpha\left|u^{*}\right| \frac{N}{N-1}\right) \mathrm{d} x=\int_{\mathbb{R}^{N}}|u|^{\beta} \zeta\left(N, \alpha|u|^{\frac{N}{N-1}}\right) \mathrm{d} x .
\end{aligned}
$$

Therefore, without loss of generality, we can take a nonnegative decreasing symmetric maximizing sequence $\left\{u_{n}(x)\right\}(n \in \mathbb{N})$ for the supremum in (1.4). Thus, for such $u_{n} \in$ $W^{1, N}\left(\mathbb{R}^{N}\right)$ with $\left\|u_{n}\right\|_{W^{1, N}\left(\mathbb{R}^{N}\right)} \leq 1$, there holds

$$
\sup _{u \in W^{1, N}\left(\mathbb{R}^{N}\right),\|u\|_{W^{1, N}\left(\mathbb{R}^{N}\right)} \leq 1} \int_{\mathbb{R}^{N}}|u|^{\beta} \zeta\left(N, \alpha u^{\frac{N}{N-1}}\right) \mathrm{d} x=\lim _{n \rightarrow \infty} \int_{\mathbb{R}^{N}}\left|u_{n}\right|^{\beta} \zeta\left(N, \alpha u_{n}^{\frac{N}{N-1}}\right) \mathrm{d} x .
$$


Since $\left\|u_{n}\right\|_{W^{1, N}\left(\mathbb{R}^{N}\right)} \leq 1$, there exists some function $\widetilde{u} \in W^{1, N}\left(\mathbb{R}^{N}\right)$ such that $u_{n} \rightarrow \widetilde{u}$ weakly in $W^{1, N}\left(\mathbb{R}^{N}\right)$ taking the subsequence if necessary. Clearly $\widetilde{u}$ is also nonnegative decreasing symmetric. In order to verify Theorem 1.2, it is sufficient to show the following result:

$$
\lim _{n \rightarrow \infty} \int_{\mathbb{R}^{N}}\left|u_{n}\right|^{\beta} \zeta\left(N, \alpha u_{n}^{\frac{N}{N-1}}\right) \mathrm{d} x=\int_{\mathbb{R}^{N}}|\widetilde{u}|^{\beta} \zeta\left(N, \alpha \widetilde{u}^{N-1}\right) \mathrm{d} x .
$$

Now we prove (4.2). Given any $R>0$, obviously we have

$$
\begin{aligned}
& \int_{\mathbb{R}^{N}}\left(\left|u_{n}\right|^{\beta} \zeta\left(N, \alpha u_{n}^{\frac{N}{N-1}}\right)-|\widetilde{u}|^{\beta} \zeta\left(N, \alpha \widetilde{u} \frac{N}{N-1}\right)\right) \mathrm{d} x \\
= & \int_{\mathbb{B}_{R}}\left(\left|u_{n}\right|^{\beta} \zeta\left(N, \alpha u_{n}^{\frac{N}{N-1}}\right)-|\widetilde{u}|^{\beta} \zeta\left(N, \alpha \widetilde{u}^{\frac{N}{N-1}}\right)\right) \mathrm{d} x \\
& \quad+\int_{\mathbb{B}_{R}^{c}}\left(\left|u_{n}\right|^{\beta} \zeta\left(N, \alpha u_{n}^{\frac{N}{N-1}}\right)-|\widetilde{u}|^{\beta} \zeta\left(N, \alpha \widetilde{u} \frac{N}{N-1}\right)\right) \mathrm{d} x \\
= & : I+I I .
\end{aligned}
$$

By the radial lemma [27], we get for any $x \in \mathbb{R}^{N} \backslash\{0\}$,

$$
\left|u_{n}(x)\right|^{N} \leq \frac{N}{\omega_{N-1}}\left\|u_{n}\right\|_{L^{N}\left(\mathbb{R}^{N}\right)}^{N} \frac{1}{|x|^{N}} .
$$

Note that

$$
\left\|u_{n}\right\|_{L^{N}\left(\mathbb{R}^{N}\right)} \leq\left\|u_{n}\right\|_{W^{1, N}\left(\mathbb{R}^{N}\right)} \leq 1
$$

Then a straightforward calculation shows

$$
\begin{aligned}
\int_{\mathbb{B}_{R}^{c}}\left|u_{n}\right|^{\beta} \zeta\left(N, \alpha u_{n}^{\frac{N}{N-1}}\right) \mathrm{d} x & =\int_{\mathbb{B}_{R}^{c}}\left(\sum_{j=N-1}^{\infty} \frac{\alpha^{j}}{j !} u_{n}^{\frac{N}{N-1} j+\beta}\right) \mathrm{d} x \\
& \leq \frac{\omega_{N-1}}{\beta} \sum_{j=N-1}^{\infty} \frac{\alpha^{j}}{j !}\left(\frac{N}{\omega_{N-1}}\right)^{\frac{1}{N-1} j+\frac{\beta}{N}} \frac{1}{R^{\frac{N}{N-1} j+\beta-N}} .
\end{aligned}
$$

It follows that

$$
\lim _{R \rightarrow \infty} \lim _{n \rightarrow \infty} \int_{\mathbb{B}_{R}^{c}}\left|u_{n}\right|^{\beta} \zeta\left(N, \alpha u_{n}^{\frac{N}{N-1}}\right) \mathrm{d} x=0
$$

With a similar consideration, we can easily get

$$
\lim _{R \rightarrow \infty} \lim _{n \rightarrow \infty} \int_{\mathbb{B}_{R}^{c}}|\widetilde{u}|^{\beta} \zeta\left(N, \alpha \widetilde{u}^{N-1}\right) \mathrm{d} x=0 .
$$

Combining (4.4) and (4.5), we obtain

$$
\lim _{R \rightarrow \infty} \lim _{n \rightarrow \infty} I I=0 .
$$


Now we estimate $I$. To see this, We denote for all $t \geq 0$,

$$
f(t)=t^{\beta} \zeta\left(N, \alpha t^{\frac{N}{N-1}}\right) \text {. }
$$

We obtain by using mean value theorem,

$$
\begin{aligned}
& I \leq\left|\int_{\mathbb{B}_{R}}\left(\left|u_{n}\right|^{\beta} \zeta\left(N, \alpha u_{n}^{\frac{N}{N-1}}\right)-|\widetilde{u}|^{\beta} \zeta\left(N, \alpha \widetilde{u}^{\frac{N}{N-1}}\right)\right) \mathrm{d} x\right| \\
& \leq \beta \int_{\mathbb{B}_{R}} \eta^{\beta-1} \zeta\left(N, \alpha \eta^{\frac{N}{N-1}}\right)\left|u_{n}-\widetilde{u}\right| \mathrm{d} x \\
& \quad+\frac{\alpha N}{N-1} \int_{\mathbb{B}_{R}} \eta^{\beta} \zeta^{\prime}\left(N, \alpha \eta^{\frac{N}{N-1}}\right) \eta^{\frac{1}{N-1}}\left|u_{n}-\widetilde{u}\right| \mathrm{d} x \\
& =\beta \int_{\mathbb{B}_{R}} \eta^{\beta-1} \zeta\left(N, \alpha \eta^{\frac{N}{N-1}}\right)\left|u_{n}-\widetilde{u}\right| \mathrm{d} x \\
& \quad+\frac{\alpha N}{N-1} \int_{\mathbb{B}_{R}} \eta^{\frac{1}{N-1}+\beta} \zeta\left(N, \alpha \eta^{\frac{N}{N-1}}\right)\left|u_{n}-\widetilde{u}\right| \mathrm{d} x \\
& \quad+\frac{\alpha^{N-1} N}{(N-1) !} \int_{\mathbb{B}_{R}} \eta^{N-1+\beta}\left|u_{n}-\widetilde{u}\right| \mathrm{d} x \\
& =: I_{1}+I_{2}+I_{3} .
\end{aligned}
$$

Here $\eta$ is nonnegative and lies between $u_{n}$ and $\tilde{u}$. Choose $p_{2}>1$ and $p_{2}^{\prime}>1$ respectively such that $\alpha p_{2}<\alpha_{N}$ and $\alpha p_{2}^{\prime}<\alpha_{N}$. By the Hölder inequality and Lemma 2.1, we have

$$
\begin{aligned}
& I_{2} \leq \frac{\alpha N}{N-1} \int_{\mathbb{B}_{R}} u_{n}^{\frac{1}{N-1}+\beta} \zeta\left(N, \alpha u_{n}^{\frac{N}{N-1}}\right)\left|u_{n}-\widetilde{u}\right| \mathrm{d} x \\
& \quad+\frac{\alpha N}{N-1} \int_{\mathbb{B}_{R}} \widetilde{u}^{\frac{1}{N-1}}+\beta \zeta\left(N, \alpha \widetilde{u} \frac{N}{N-1}\right)\left|u_{n}-\widetilde{u}\right| \mathrm{d} x \\
& \leq \alpha_{N} N\left(\int_{\mathbb{B}_{R}} u_{n}^{\left(\frac{1}{N-1}+\beta\right) p_{1}} \mathrm{~d} x\right)^{\frac{1}{p_{1}}}\left(\int_{\mathbb{B}_{R}} \zeta\left(N, \alpha p_{2} u_{n}^{\frac{N}{N-1}}\right) \mathrm{d} x\right)^{\frac{1}{p_{2}}}\left(\int_{\mathbb{B}_{R}}\left|u_{n}-\widetilde{u}\right|^{p_{3}} \mathrm{~d} x\right)^{\frac{1}{p_{3}}} \\
& \quad+\alpha_{N} N\left(\int_{\mathbb{B}_{R}} \widetilde{u}^{\left(\frac{1}{N-1}+\beta\right) p_{1}^{\prime}} \mathrm{d} x\right)^{\frac{1}{p_{1}^{\prime}}}\left(\int_{\mathbb{B}_{R}} \zeta\left(N, \alpha p_{2}^{\prime} \widetilde{u} \frac{N}{N-1}\right) \mathrm{d} x\right)^{\frac{1}{p_{2}^{\prime}}}\left(\int_{\mathbb{B}_{R}}\left|u_{n}-\widetilde{u}\right|^{p_{3}^{\prime}} \mathrm{d} x\right)^{\frac{1}{p_{3}^{\prime}}} \\
& \leq C_{1}\left\|u_{n}-\widetilde{u}\right\|_{L^{p_{3}\left(\mathbb{B}_{R}\right)}}+C_{2}\left\|u_{n}-\widetilde{u}\right\|_{L^{p_{3}^{\prime}}\left(\mathbb{B}_{R}\right)^{\prime}}
\end{aligned}
$$

where $1 / p_{1}+1 / p_{2}+1 / p_{3}=1,1 / p_{1}^{\prime}+1 / p_{2}^{\prime}+1 / p_{3}^{\prime}=1$. $C_{1}$ is a constant depending only on $N, R, \alpha, \beta, p_{1}, p_{2}$; while $C_{2}$ is a constant depending only on $N, R, \alpha, \beta, p_{1}^{\prime}, p_{2}^{\prime}$. Letting $n \rightarrow \infty$ and next $R \rightarrow \infty$, then

$$
\lim _{R \rightarrow \infty} \lim _{n \rightarrow \infty} I_{2}=0 .
$$

Meanwhile, we have by the Hölder inequality,

$$
I_{3} \leq C\left(\int_{\mathbb{B}_{R}}\left(u_{n}+\widetilde{u}\right)^{(N-1+\beta) s} \mathrm{~d} x\right)^{1 / s}\left(\int_{\mathbb{B}_{R}}\left|u_{n}-\widetilde{u}\right|^{t} \mathrm{~d} x\right)^{1 / t} \leq C\left\|u_{n}-\widetilde{u}\right\|_{L^{t}\left(\mathbb{B}_{R}\right)},
$$


where $1 / s+1 / t=1$ and $C$ is a constant that depends only on $N, R, \alpha, \beta, s$. Then we have

$$
\lim _{R \rightarrow \infty} \lim _{n \rightarrow \infty} I_{3}=0 .
$$

In order to estimate $I_{1}$, we distinguish two cases as follows.

Case 1: $\beta \geq 1$. If $\beta \geq 1$. An obvious analog of (4.8) is

$$
\lim _{R \rightarrow \infty} \lim _{n \rightarrow \infty} I_{1}=0 .
$$

Combining (4.7)-(4.10), we get

$$
\lim _{R \rightarrow \infty} \lim _{n \rightarrow \infty} I=\lim _{R \rightarrow \infty} \lim _{n \rightarrow \infty}\left(I_{1}+I_{2}+I_{3}\right)=0 .
$$

This together with (4.3) and (4.6) leads to

$$
\lim _{n \rightarrow \infty} \int_{\mathbb{R}^{N}}\left(\left|u_{n}\right|^{\beta} \zeta\left(N, \alpha u_{n}^{\frac{N}{N-1}}\right)-|\widetilde{u}|^{\beta} \zeta\left(N, \alpha \widetilde{u} \frac{N}{N-1}\right)\right) \mathrm{d} x=0,
$$

which is equivalent to (4.2).

Case 2: $0<\beta<1$. The difficulty in this case lies in the estimate of $I_{1}$ in (4.7). Since $-1<\beta-1<0$, the integrability of $u^{\beta-1}$ is not clear. The Hö inequality can not be applied directly. This motivates us to define the function by

$$
g(t)=t^{\beta-1} \zeta\left(N, \alpha t^{\frac{N}{N-1}}\right)=\sum_{j=N-1}^{\infty} \frac{\alpha^{j}}{j !} t^{\frac{N}{N-1} j+\beta-1},
$$

for any $t \geq 0$. One checks easily that $g(t)$ is an increasing function. Moreover, for any nonnegative function $u \in W^{1, N}\left(\mathbb{R}^{N}\right)$ with $\|u\|_{W^{1, N}\left(\mathbb{R}^{N}\right)} \leq 1$,

$$
\begin{aligned}
g(u) & =u^{N+\beta-1}\left(\sum_{j=N-1}^{\infty} \frac{\alpha^{j}}{j !} u^{\frac{N}{N-1}}(j-N+1)\right) \\
& =u^{N+\beta-1}\left(\chi_{\{u>1\}}+\chi_{\{u \leq 1\}}\right)\left(\sum_{j=N-1}^{\infty} \frac{\alpha^{j}}{j !} u^{\frac{N}{N-1}(j-N+1)}\right) \\
& \leq u^{N+\beta-1}\left(\sum_{j=N-1}^{\infty} \frac{\alpha^{j}}{j !} u^{\frac{N}{N-1} j}\right)+\sum_{j=N-1}^{\infty} \frac{\alpha^{j}}{j !} \\
& =u^{N+\beta-1} \zeta\left(N, \alpha u^{\frac{N}{N-1}}\right)+\sum_{j=N-1}^{\infty} \frac{\alpha^{j}}{j !},
\end{aligned}
$$

where $\chi_{\{u>1\}}$ denotes the characteristic function of set $\left\{x \in \mathbb{R}^{N}: u(x)>1\right\}$. Take $p>1$ such that $\alpha p<\alpha_{N}$. In view of (4.11), we have by the Minkowski inequality and Lemma 2.1,

$$
\left(\int_{\mathbb{B}_{R}} g(u)^{p} \mathrm{~d} x\right)^{1 / p} \leq\left(\int_{\mathbb{B}_{R}} u^{(N+\beta-1) p} \zeta\left(N, \alpha p u^{\frac{N}{N-1}}\right) \mathrm{d} x\right)^{1 / p}+\left(\sum_{j=N-1}^{\infty} \frac{\alpha^{j}}{j !}\right)\left|\mathbb{B}_{R}\right|^{1 / p} .
$$


Since $(N+\beta-1) p>1$, we conclude that the first term in the above inequality is finite by virtue of the analysis of Case 1. Obviously, the second term is also bounded. Therefore $g(u) \in L^{p}\left(\mathbb{B}_{R}\right)$ for some $p>1$.

By calculation, one has

$$
\begin{aligned}
I_{1} & =\int_{\mathbb{B}_{R} \cap\left\{u_{n} \leq 1\right\}} \beta g(\eta)\left|u_{n}-\widetilde{u}\right| \mathrm{d} x+\int_{\mathbb{B}_{R} \cap\left\{u_{n}>1\right\}} \beta g(\eta)\left|u_{n}-\widetilde{u}\right| \mathrm{d} x \\
& \leq \int_{\mathbb{B}_{R} \cap\left\{u_{n} \leq 1\right\}}\left(g\left(u_{n}\right)+g(\widetilde{u})\right)\left|u_{n}-\widetilde{u}\right| \mathrm{d} x+\int_{\mathbb{B}_{R} \cap\left\{u_{n}>1\right\}}\left(g\left(u_{n}\right)+g(\widetilde{u})\right)\left|u_{n}-\widetilde{u}\right| \mathrm{d} x \\
& \leq \int_{\mathbb{B}_{R} \cap\left\{u_{n} \leq 1\right\}}(g(1)+g(\widetilde{u}))\left|u_{n}-\widetilde{u}\right| \mathrm{d} x+\int_{\mathbb{B}_{R} \cap\left\{u_{n}>1\right\}}\left(\zeta\left(N, \alpha u_{n}^{\frac{N}{N-1}}\right)+g(\widetilde{u})\right)\left|u_{n}-\widetilde{u}\right| \mathrm{d} x .
\end{aligned}
$$

Note that $g(\widetilde{u}) \in L^{p}\left(\mathbb{B}_{R}\right)$ for some $p>1$. The first term tends to 0 as $n \rightarrow \infty$ thanks to the Hö inequalitythe Minkowski inequality and the Sobolev embedding theorem. Again using these inequalities together with inequality (1.2) and Lemma 2.1, we conclude that the second term tends to 0 as $n \rightarrow \infty$. Therefore

$$
\lim _{R \rightarrow \infty} \lim _{n \rightarrow \infty} I_{1}=0 .
$$

This together with (4.8) and (4.9) implies

$$
\lim _{R \rightarrow \infty} \lim _{n \rightarrow \infty} I=0 .
$$

Hence, (4.2) follows from (4.3), (4.6) and (4.12) in this case.

Combining the above two cases, we conclude that (4.2) holds. It follows from (4.1) and (4.2) that $\widetilde{u}$ attains the supremum in (1.4).

Finally, we prove $\|\widetilde{u}\|_{W^{1, N}\left(\mathbb{R}^{N}\right)}=1$. Suppose not. Clearly, $\widetilde{u} \not \equiv 0$. Assume $\|\widetilde{u}\|_{W^{1, N}\left(\mathbb{R}^{N}\right)}<1$. We set $\bar{u}=\widetilde{u} /\|\widetilde{u}\|_{W^{1, N}\left(\mathbb{R}^{N}\right)}$, then $\|\bar{u}\|_{W^{1, N}\left(\mathbb{R}^{N}\right)}=1$. Moreover, we obtain

$$
\begin{gathered}
\sup _{u \in W^{1, N}\left(\mathbb{R}^{N}\right),\|u\|_{W^{1, N}\left(\mathbb{R}^{N}\right)} \leq 1} \int_{\mathbb{R}^{N}}|u|^{\beta} \zeta\left(N, \alpha|u|^{\frac{N}{N-1}}\right) \mathrm{d} x \\
\geq \int_{\mathbb{R}^{N}}|\bar{u}|^{\beta} \zeta\left(N, \alpha|\bar{u}|^{\frac{N}{N-1}}\right) \mathrm{d} x>\int_{\mathbb{R}^{N}}|\widetilde{u}|^{\beta} \zeta\left(N, \alpha|\widetilde{u}|^{\frac{N}{N-1}}\right) \mathrm{d} x,
\end{gathered}
$$

which contradicts the fact that $\widetilde{u}$ attains the supremum in (1.4). Thus, $\|\widetilde{u}\|_{W^{1, N}\left(\mathbb{R}^{N}\right)}=1$. Therefore $\widetilde{u}$ is the desired extremal function. The proof of Theorem 1.2 is completely finished.

\section{Acknowledgments}

The author would like to thank referee for valuable suggestions. The work is supported by the Natural Science Foundation of the Education Department of Anhui Province (KJ20 -16A641). 


\section{References}

[1] Moser J., A sharp form of an inequality by N. Trudinger. Indiana. Univ. Math. J., 20 (1971), 1077-1091.

[2] Peetre J., Espaces d'interpolation et theoreme de Soboleff. Ann. Inst. Fourier (Grenoble), 16 (1966), 279-317.

[3] Pohozaev S., The Sobolev embedding in the special case $p l=n$. Proceedings of the Technical Scientific Conference on Advances of Scientific Research 1964-1965, Mathematics Sections, Moscov. Energet. Inst., Moscow, (1965), 158-170.

[4] Trudinger N., On embeddings into Orlicz space and some applications. J. Math. Mech., 17 (1967), 473-483.

[5] Yudovich V. I., Some estimates connected with integral operators and with solutions of elliptic equations. Sov. Math. Docl., 2 (1961), 746-749.

[6] Cao D., Nontrivial solution of semilinear elliptic equation with critical exponent in $\mathbb{R}^{2}$. Comm. Partial Differential Equations, 17 (1992), 407-435.

[7] Panda R., Nontrivial solution of a quasilinear elliptic equation with critical growth in $\mathbb{R}^{n}$. Proc. Indian Acad. Sci. Math. Sci., 105 (1995), 425-444.

[8] do Ó J. M., N-Laplacian equations in $R^{N}$ with critical growth. Abstr. Appl. Anal., 2 (1997), 301-315.

[9] Adachi S., Tanaka K., Trudinger type inequalities in $\mathbb{R}^{N}$ and their best exponents. Proc. Amer. Math. Soc., 128 (1999), 2051-2057.

[10] Ruf B., A sharp Trudinger-Moser type inequality for unbounded domains in $\mathbb{R}^{2}$. J. Funct. Anal., 219 (2005), 340-367.

[11] Li Y., Ruf B., A sharp Trudinger-Moser type inequality for unbounded domains in $\mathbb{R}^{N}$. Indiana. Univ. Math. J., 57 (2008), 451-480.

[12] Adimurthi, Yang Y., An interpolation of Hardy inequality and Trudinger-Moser inequality in $\mathbb{R}^{N}$ and its applications. International Mathematics Research Notices, 13 (2010), 2394-2426.

[13] Ishiwata M., Existence and nonexistence of maximizers for variational problems associated with Trudinger-Moser type inequalities in $\mathbb{R}^{N}$. Math. Ann., 351 (2011), 781-804.

[14] Carleson L., Chang A., On the existence of an extremal function for an inequality of J. Moser. Bull. Sci. Math., 110 (1986), 113-127.

[15] Flucher M., Extremal functions for Trudinger-Moser inequality in 2 dimensions. Comment. Math. Helv., 67 (1992), 471-497.

[16] Lin K., Extremal functions for Moser's inequality. Trans. Amer. Math. Soc., 348 (1996), 26632671.

[17] Li Y., Moser-Trudinger inequality on compact Riemannian manifolds of dimension two. J. Part. Diff. Equations, 14 (2001), 163-192.

[18] Yang Y., A sharp form of the Moser-Trudinger inequality on a compact Riemannian surface. Trans. Amer. Math. Soc., 359 (2007), 5761-5776.

[19] Yang Y., Extremal functions for Trudinger-Moser inequalities of Adimurthi-Druet type in dimension two. J. Differential Equations, 258 (2015), 3161-3193.

[20] Yang Y., A Trudinger-Moser inequality on a compact Riemannian surface involving Gaussian curvature. J. Geom. Anal., 26 (2016), 2893-2913.

[21] Lu G., Yang Y., The sharp constant and extremal functions for Moser-Trudinger inequalities involving $L^{p}$ norms. Discrete and Continuous Dynamical Systems, 25 (2009), 963-979.

[22] Wang G., Ye D., A Hardy-Moser-Trudinger inequality. Adv. Math., 230 (2012), 294-320. 
[23] Yang Y., Zhu X., An improved Hardy-Trudinger-Moser inequality. Ann. Global Anal. Geom., 49 (2016), 23-41.

[24] Yang Y., Zhu X., Blow-up analysis concerning singular Trudinger-Moser inequalities in dimension two. J. Funct. Anal. (2017), DOI: 10.1016/j.jfa.2016.12.028.

[25] Yang Y., Existence of positive solutions to quasi-linear elliptic equations with exponential growth in the whole Euclidean space. J. Funct. Anal., 262 (2012), 1679-1704.

[26] Hardy G., Littlewood J. and Polya G., Inequalities, Cambridge University Press, Cambridge, 1952.

[27] Berestycki H., Lions P. L., Nonlinear scalar field equations I. Existence of ground state. Arch. Rational Mech. Anal., 82 (1983), 313-346. 